Draft version August 30, 2018

Preprint typeset using $\mathrm{IAT}_{\mathrm{E}} \mathrm{X}$ style emulateapj v. 5/2/11

\title{
PROSPECTS FOR PROBING THE SPACETIME OF Sgr A* WITH PULSARS
}

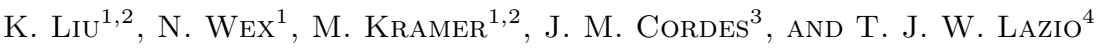 \\ ${ }^{1}$ Max-Planck-Institut für Radioastronomie, Auf dem Hügel 69, 53121, Bonn, Germany \\ ${ }^{2}$ University of Manchester, Jodrell Bank Centre for Astrophysics, Alan-Turing Building, Manchester M13 9PL, UK \\ ${ }^{3}$ Astronomy Dept., Cornell Univ., Ithaca, NY 14853, USA and \\ ${ }^{4}$ Jet Propulsion Laboratory, M/S 138-308, 4800 Oak Grove Dr., Pasadena, CA 91109, USA \\ Draft version August 30, 2018
}

\begin{abstract}
The discovery of radio pulsars in compact orbits around Sgr A* would allow an unprecedented and detailed investigation of the spacetime of the supermassive black hole. This paper shows that pulsar timing, including that of a single pulsar, has the potential to provide novel tests of general relativity, in particular its cosmic censorship conjecture and no-hair theorem for rotating black holes. These experiments can be performed by timing observations with $100 \mu$ s precision, achievable with the Square Kilometre Array for a normal pulsar at frequency above $15 \mathrm{GHz}$. Based on the standard pulsar timing technique, we develop a method that allows the determination of the mass, spin, and quadrupole moment of $\mathrm{Sgr} \mathrm{A}^{*}$, and provides a consistent covariance analysis of the measurement errors. Furthermore, we test this method in detailed mock data simulations. It seems likely that only for orbital periods below $\sim 0.3 \mathrm{yr}$ is there the possibility of having negligible external perturbations. For such orbits we expect $\mathrm{a} \sim 10^{-3}$ test of the frame dragging and $\mathrm{a} \sim 10^{-2}$ test of the no-hair theorem within 5 years, if Sgr A* is spinning rapidly. Our method is also capable of identifying perturbations caused by distributed mass around Sgr A*, thus providing high confidence in these gravity tests. Our analysis is not affected by uncertainties in our knowledge of the distance to the Galactic center, $R_{0}$. A combination of pulsar timing with the astrometric results of stellar orbits would greatly improve the measurement precision of $R_{0}$.

Subject headings: black hole physics; Galaxy: center; pulsars: general
\end{abstract}

\section{INTRODUCTION}

One of the most intriguing results of general relativity (GR) is the uniqueness theorem for the stationary black hole solutions of the Einstein-Maxwell equations (see Heusler 1998, and references therein). This uniqueness theorem states that (under certain conditions) all stationary electrovad 1 black hole spacetimes with a nondegenerate horizon are described by the Kerr-Newman metric. It implies that in GR all stationary black holes are parameterized by only three parameters: mass $(M)$, spin $(S)$ and electric charge ("black holes have no hair"). All uncharged black hole solutions are described by the Kerr metric and, therefore, uniquely determined by $M$ and $S$. Astrophysical black holes are believed to be the result of a gravitational collapse. During this collapse all the properties of the progenitor, apart from mass and spin, are radiated away by gravitational radiation while the gravitational field asymptotically approaches its stationary configuration (Price 1972a, b) . The outer spacetime of an astrophysical black hole should therefore be described by the Kerr metric Since the Kerr metric has a maximum spin at which it still exhibits an event horizon, Penrose's cosmic censorship conjecture (CCC) within GR (Penrose 1979) requires the dimensionless spin

\footnotetext{
${ }^{1}$ Electrovac spacetimes are the solutions of the Einstein-Maxwell equations.

2 Strictly speaking, this is only true for a certain approximation since, to some extent, astrophysical black holes will be influenced by nearby masses (accretion, orbiting objects). We will address this issue for Sgr A* in this paper at the end of the discussion Section.
}

parameter $\chi$ to satisfy

$$
\chi \equiv \frac{c}{G} \frac{S}{M^{2}} \leq 1
$$

A measured value for $\chi$ that exceeds 1 would pose a serious problem for our understanding of spacetime, since this would indicate that either GR is wrong or that a region may be visible to the outside universe, where our present understanding of gravity and spacetime breaks down.

As a result of the no-hair theorem, all higher multipole moments $(l \geq 2)$ of the gravitational field of an astrophysical black hole can be expressed as a function of $M$ and $S$ (Hansen 1974). In particular, the dimensionless quadrupole moment $q$ satisfies the relation

$$
q \equiv \frac{c^{4}}{G^{2}} \frac{Q}{M^{3}}=-\chi^{2} .
$$

A measurement of the quadrupole moment, in combination with a mass and a spin measurement, would therefore provide a test of the no-hair theorem for Kerr black holes.

Some of the clearest evidence for the existence of black holes comes from the monitoring of $\sim 30$ stellar orbits in the center of our Galaxy (Schödel et al. 2002; Ghez et al. 2008; Gillessen et al. 2009), where the shortest orbital period, $P_{\mathrm{b}}$, is 16 years. Known by its radio nomenclature of Sgr A*, current estimates put the mass of this black hole to around $4 \times 10^{6} M_{\odot}$. A black hole of that size at a distance of $8 \mathrm{kpc}$ is an ideal laboratory for blackhole physics, strong-field gravity and, in particular, a test of the no-hair theorem for Kerr black holes (e.g. Psaltis 
2008; Johannsen \& Psaltis 2011). It has been shown by Will (2008) that the discovery of stars in highly eccentric $(e \sim 0.9)$ orbits very close to $\operatorname{Sgr} \mathrm{A}^{*}\left(P_{\mathrm{b}} \lesssim 0.1 \mathrm{yr}\right)$ could be used to test the general relativistic no-hair theorem. This experiment requires an astrometric precision at the level of $10 \mu \mathrm{as}$, which seems achievable with the upcoming infrared-astrometry experiments, such as GRAVITY (Eisenhauer et al. 2009). At a distance of $8 \mathrm{kpc}$, an angle of $10 \mu$ as corresponds to a length scale of $\sim 10^{7} \mathrm{~km}$.

On the other hand, if close-in pulsars could be found and tracked in their orbits, even for those with poor timing precision the time-of-arrival (TOA) for a (integrated) pulsar signal can be measured with an uncertainty of a few milliseconds, corresponding to a light-travel distance of $\sim 10^{3} \mathrm{~km}$. Moreover, a phase-connected solution with an appropriate timing model allows a determination of the pulsar orbit, which provides even more precision. Hence, as shown by Wex \& Kopeikin (1999) and Kramer et al. (2004), a pulsar in orbit around the supermassive black hole in the Galactic center (GC) would provide an ideal probe to measure the mass, the spin, and the quadrupole moment of $\mathrm{Sgr} \mathrm{A}^{*}$, and consequently test the no-hair theorem for Kerr black holes. In their discussion on "bumpy black holes", Vigeland \& Hughes (2010) suggest that a pulsar in orbit around a black hole could be used for mapping its multipole moment structure. In a recent publication Angélil et al. (2010) have discussed the importance of frame dragging and the quadrupole moment for stars and pulsars in orbit around Sgr A*, based on numerical integration of geodesics in a Kerr spacetime. For pulsars, however, the results in this paper are only indicative 3 Wang et al. (2009a,b) have shown that if a pulsar is found in a very close orbit around Sgr A* (e.g. $P_{\mathrm{b}} \lesssim 1$ day), observers at the Earth can receive additional pulses travelling along a path that is strongly bent by the gravitational potential of the black hole. The exploitation of this information would provide unique constraints on the strongly curved spacetime geometry near Sgr A*.

In this paper we will demonstrate how the mass, the spin (magnitude and orientation) and the quadrupole moment of Sgr A* can be determined from timing a pulsar in a sufficiently tight orbit around the supermassive black hole. Our analysis is based on simulated TOAs and a timing model that allows for a phase-connected solution, consistently accounting for the relativistic effects in the motion of the pulsar and the propagation of the radio signals. Based on this, we can determine the expected precision for the individual parameters while accounting for all the correlations between the parameters. In all discussions and simulations we focus on the leading order in the individual effects of interest. We are well aware that in an actual timing model for orbits close to Sgr $\mathrm{A}^{*}$ we need to account for higher order effects in

\footnotetext{
3 The Angélil et al. (2010) results for a pulsar are not derived from a consistent covariance analysis based on a timing model that incorporates all the relevant effects simultaneously. Moreover, pulsar timing is treated as a radial velocity measurement experiment, which is incorrect. In fact, pulsar timing makes use of phaseconnected solutions for the rotational phase of a pulsar leading to a precision in the parameter estimations that can be several orders of magnitude better (Lorimer \& Kramer 2005). Also, the timing precision assumed by Angélil et al. (2010) seems too optimistic for a GC pulsar, as will become clear from the analysis presented in this paper.
}

the orbital motion and signal propagation. For many of the effects discussed below, higher order terms have already been calculated (e.g. Damour \& Schäfer 1988; Wex 1995; Kopeikin 1997; Königsdörffer \& Gopakumar 2005). However, to estimate the expected precision and covariances in the parameter determination, it is sufficient to use a timing model that combines just the leading terms of all contributions relevant here. The second order terms contribute at $\mathrm{a} \sim \beta_{\mathrm{O}}^{2}$ level, where $\beta_{\mathrm{O}}$ is the orbital velocity parameter introduced by Damour \& Taylor (1992). For a test particle in orbit around a mass $M$

$$
\begin{aligned}
\beta_{\mathrm{O}} & =\left(\frac{2 \pi G M}{c^{3} P_{\mathrm{b}}}\right)^{1 / 3} \\
& \simeq 0.0158\left(\frac{M}{4 \times 10^{6} M_{\odot}}\right)^{1 / 3}\left(\frac{P_{\mathrm{b}}}{1 \mathrm{yr}}\right)^{-1 / 3} .
\end{aligned}
$$

As an example, for an orbital period of $0.1 \mathrm{yr}$ we find $\beta_{\mathrm{O}}^{2} \approx 10^{-3}$. At this point it is worth mentioning, that for the most relativistic binary pulsar, the double pulsar, one finds $\beta_{\mathrm{O}}^{2} \simeq 4.3 \times 10^{-6}$ (Kramer \& Wex 2009), which nicely illustrates how much more relativistic a pulsar in a $P_{\mathrm{b}} \lesssim 1$ yr orbit around Sgr $\mathrm{A}^{*}$ would be.

Previous studies suggest that about 1000 pulsars can be expected to be orbiting $\operatorname{Sgr} \mathrm{A}^{*}$ with periods less than 100 years (Cordes \& Lazio 1997; Genzel et al. 2003; Pfahl \& Loeb 2004), and some of them may be associated with remnants of the observed S-star population in the neighborhood. Deep pulsar searches towards the GC region were already conducted with a few radio telescopes (Effelsberg, Green Bank, Parkes) at frequencies up to $15 \mathrm{GHz}$ (e.g. Kramer et al. 2000; Macquart et al. 2010). Five pulsars were found no more than 200 pc away from Sgr A* Johnston et al. 2006; Deneva et al. 2009, Kramer et al. private communication), which is consistent with the estimated large pulsar population within that region. However, they are not close enough to the supermassive black hole to probe its gravitational field. In this paper we will focus on timing observations of such pulsars, and show how far they could take us in probing the gravitational field of $\mathrm{Sgr} \mathrm{A}^{*}$, provided the system is found to be sufficiently free of external perturbations. The main purpose therefore is the development of the methodology, and the estimation of its potentials in testing the Kerr nature of Sgr A* based on mock data simulations. For further elaboration on either the existence of pulsars in close orbits around Sgr A* or the search for them we refer to the rich literature (Lorimer \& Kramer 2005) and future work in progress. In Section 2 we discuss the expected timing precision and the orbital periods required for our measurements. Section 3 presents the various relativistic effects that can be used to determine the mass of $\mathrm{Sgr} \mathrm{A}^{*}$ and, based on simulations, the expected precision in the mass measurement. In Section 4 we show how the spin can be extracted from the timing measurement, how this information can be used to test the CCC, and how the presence of a distributed mass in the vicinity of Sgr A* would affect this measurement. Section 5 provides the details on the quadrupole measurement and the no-hair theorem test. In Section 6

4 The result from the GC survey by Macquart et al. (2010) indicated that the actual number of such pulsars may be less. 
we summarize and discuss our findings.

\section{TIMING A PULSAR IN ORBIT AROUND Sgr A*}

Pulsar timing involves measuring the TOAs of a pulsar's pulses and monitoring them on a timescale of years (e.g. Tavlor 1992). The precision of TOA measurements of young pulsars near the GC, by future telescopes, will mainly be limited by three effects that have significantly different dependencies on observing frequency: firstly the signal-to-noise ratio of the measured pulses, secondly the pulse phase jitter intrinsic to the pulsar, and thirdly the changes in pulse shape caused by interstellar scintillation (Cordes \& Shannon 2010; Liu et al. 2011). The first effect is independent of frequency under our assumption that the pulse width does not change with frequency. In reality, the pulse width does evolve but that is secondary to the overall timing error. The second and third effects are strongly frequency dependent, due to the steep pulsar spectrum and the pulse broadening caused by the large amount of scattering from the high electron density in the ionized gas near the GC. The strong dependence of scattering on frequency $\left(\propto f^{-4}\right.$, see the next paragraph) implies that observations need to be made at much higher frequencies than are typically used for pulsar timing.

There have been previous studies on optimizing the observational frequency for the purpose of pulsar searches towards the central parsec region (Cordes \& Lazio 1997; Macquart et al. 2010). Fig. 1 shows the estimated timing precision for a canonical pulsar near Sgr A* as a function of the observing frequency. The calculation of the achievable TOA uncertainty $\sigma_{\text {TOA }}$ accounts for three contributions:

$$
\sigma_{\mathrm{TOA}}^{2}=\sigma_{\mathrm{rn}}^{2}+\sigma_{J}^{2}+\sigma_{\text {scint }}^{2} .
$$

Here $\sigma_{\mathrm{rn}}, \sigma_{J}$, and $\sigma_{\text {scint }}$ represent the uncertainties contributed by radiometer noise, pulse phase jitter and interstellar scintillation, respectively, which can be calculated by following e.g. Cordes \& Shannon (2010). Specifically, we use a spin period $P=0.5 \mathrm{~s}$, an intrinsic pulse width $W_{i}=10 \mathrm{~ms}$, and a period-averaged flux density $S_{1400}=$ $1 \mathrm{mJy}$ at $1.4 \mathrm{GHz}$. For a $100-\mathrm{m}$ diameter dish and the SKA, we use a gain of $2 \mathrm{~K} \mathrm{Jy}^{-1}, 100 \mathrm{~K} \mathrm{Jy}^{-1}$, and an integration time of $4 \mathrm{hr}, 1 \mathrm{hr}$, respectively. Two different spectral indices of the pulsar flux density, which typifies many of these measured for pulsars (Maron et al. 2000), are used in our calculations. The scattering time scale is estimated to be $\tau_{\text {scat }} \approx 2.3 \times 10^{6} \mathrm{~ms}$ at $1 \mathrm{GHz}$, as derived from the observed scattering diameter of $\mathrm{Sgr} \mathrm{A}^{*}$ and the estimated location of the scattering material along the line of sight, the latter as incorporated in the NE2001 model $(\ell=b=0$ and $D=8.5 \mathrm{kpc}$, Cordes \& Lazio 2002). For this large amount of scattering, we use a scaling of $\tau_{\text {scat }} \propto f^{-4}$ (e.g. Löhmer et al. 2001) rather than the often used Kolmogorov scaling $\tau_{\text {scat }} \propto f^{-4.4}$ (e.g. Rickett 1990), because the dominant length scale is less than the inner scale of the wavenumber spectrum for the electron density. Note that all potential pulsars with close orbits of interest for the GR tests will be seen along essentially the same line of sight as $\mathrm{Sgr} \mathrm{A}^{*}$, so one can assume that their lines of sight will have the same scattering characteristics. The system temperature (e.g. $\simeq 40 \mathrm{~K}$ at $15 \mathrm{GHz}$ ) is calculated by summing the radio background, receiver temperature and emission of the atmosphere. It clearly follows from Fig. 1, that with a radio telescope like
SKA TOA uncertainties of below $100 \mu$ s seem likely for an observational frequency above $15 \mathrm{GHz}$, similar to the result of optimized searching frequency. A detection of MSPs in the Galactic centre is unlikely (Cordes \& Lazio 1997; Macquart et al. 2010), so they are not considered in the following simulations. However, we will show that the black hole properties can already be extracted by finding and timing a relatively slow pulsar. If a MSP were to be found after all, the experiment may reach a correspondingly higher precision.

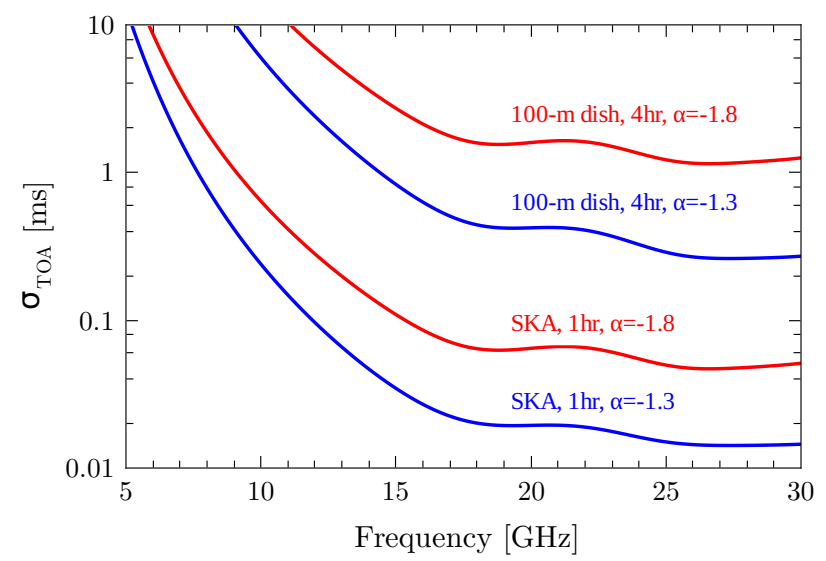

FIG. 1. - Predicted TOA measurement precision of a young pulsar near the GC for two different spectral indices $\alpha$. The curves take into account pulse phase jitter intrinsic to the pulsar, pulse broadening from scattering along the entire line of sight, and from the finite number of scintles included in the measurement. Scattering is dominated by a region of high plasma density that surrounds the GC. We assumed a four hour integration time using a 100-meter radio telescope and a one hour integration time using an SKA-like telescope, both with a highest operating frequency of $30 \mathrm{GHz}$ and a bandwidth of $1 \mathrm{GHz}$. It is found that observational frequencies above $15 \mathrm{GHz}$ favors pulsar timing observation, where $100 \mu \mathrm{s}$ TOA precision seems achievable, in particular with the SKA. The parameters used for this calculations can be found in the text.

Precision of long-term timing of a young pulsar is often limited by irregularities of the pulsar's rotation, supposedly associated with either the internal super-fluid flux (e.g. Melatos \& Warszawski 2009), or external magnetic field activity (e.g. Lvne et al. 2010). The amplitude of the low-frequency noise resulting from these instabilities varies from about $10 \mu \mathrm{s}$ to $100 \mathrm{~ms}$ (Hobbs et al. 2010), and in some cases the noise can be modeled by following the approach proposed by Lyne et al. (2010) to improve the timing precision by order of magnitudes. Consequently, a TOA precision of $100 \mu$ s is a reasonable fiducial value, which we will use in our simulations below.

Although the purpose of this paper is to discuss potential gravity tests with a pulsar in orbit around Sgr A*, provided the system is found to be sufficiently clean, we nevertheless would like to complete this Section with a brief discussion on possible effects that could complicate or even spoil these tests. Merritt et al. (2010) and Sadeghian \& Will (2011) have shown that for orbits with an orbital period $P_{\mathrm{b}}$ larger than $0.1 \mathrm{yr}$, it becomes likely that the distribution of stars in the vicinity causes "external" perturbations of the orbital motion of the pulsar and prevent a clean test of the no-hair theorem or even a measurement of the Lense-Thirring ef- 
fect. In order to evaluate the significance of the perturbation, following the analysis of Merritt et al. (2010), in Fig. 2 the relation of precessional timescale against orbital size is presented for four different contributions: the pericenter advance, the frame dragging effect, the black-hole quadrupole, and the surrounding mass distribution. Here we assume $10^{3}$ (the highest number applied in Merritt et al. 2010) one solar mass objects isotropically distributed within $1 \mathrm{mpc}$ around Sgr A*. Similar to Merritt et al. (2010) we do not consider the influence of objects outside the central $1 \mathrm{mpc}$ region. One can see that for wide orbits $\left(P_{\mathrm{b}} \sim 10 \mathrm{yr}\right)$ the pericenter advance is still significantly larger than the precession caused by external perturbations. This suggests that for orbital periods less than about 10 years the measured $\dot{\omega}$ can be used to tightly constrain the black hole mass. The frame dragging will be dominant over the stellar noise if the orbital period is less than 0.5 years, while only for orbital periods $\lesssim 0.1 \mathrm{yr}$ the (secular) contribution of the quadrupole moment is expected to be significantly above the external perturbation. We note that the assumptions applied to calculate the precessional timescale by stellar perturbation may not be secure, as the actual stellar components and mass distributions within the central pc (especially the central mpc) are still not fully understood. For instance, the precessional torque could be larger if there exists a high fraction of massive objects near Sgr A* due to mass segregation (O'Leary et al. 2009; Keshet et al. 2009; Kocsis \& Tremaine 2011), or a significant anisotropy in the distribution of the surrounding masses. In fact, it has been argued by Merritt et al. (2010) that a high fraction of $10 M_{\odot}$ black holes in this region would make astrometric tests of general relativity problematic at all radii. Concerning pulsars, however, as will be discussed in Sections 4.3 and 5.2 , the gravitomagnetic and quadrupolar field of Sgr A* will result in unique features in the timing residuals, which can be tracked well with high precision timing observations, and one can still expect to be able to extract the Sgr A* spin and quadrupole moment from the timing data, to some extent. This however depends strongly on the details of the external perturbations, which only will be known once a pulsar is discovered in that region.

\section{MASS MEASUREMENT}

The current best estimate for the mass of Sgr A* gives $4.30 \pm 0.20_{(\text {stat })} \pm 0.30_{(\text {sys })} \times 10^{6} M_{\odot} \quad$ Gillessen et al. 2009)5. The proposed method has the potential to improve the measurement accuracy by a factor of $\sim 10^{5}$. This is possible pulsar timing. Indeed, the most precise mass measurements for stars (other than the Sun) come from pulsar timing observations (Jacoby et al. 2005; Freire et al. 2011). Those are achieved in binary pulsar systems, where in addition to the Keplerian parameters one can determine a set of post-Keplerian (PK) parameters as theory independent relativistic corrections. In any theory of gravity the PK parameters are functions of the two a priori unknown masses of the system, which can be determined once two PK parameters have been obtained (see Damour \& Taylor 1992, for definitions of the Keplerian and PK parameters). Since in our case

5 The main uncertainty is from the limited knowledge of the distance to the GC.

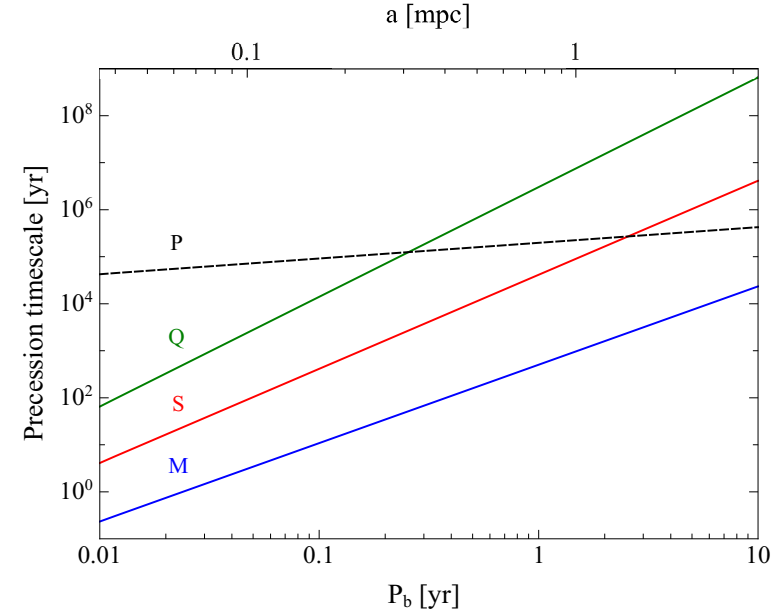

FIG. 2.- Timescales of secular orbital precession for a pulsar in orbit around $\mathrm{Sgr} \mathrm{A}^{*}$ as a function of orbital period $P_{\mathrm{b}}$ (semi-major axis $a$ ). The letters $\mathrm{M}, \mathrm{S}, \mathrm{Q}$ and $\mathrm{P}$ stand for the contribution by the mass monopole (pericenter advance), the spin (frame dragging), the quadrupole moment, and stellar perturbation, respectively. Here we assume an orbital eccentricity of 0.5 and $10^{3}$ one solar mass objects within $1 \mathrm{mpc}$ around Sgr A*. As a comparison, the Schwarzschild radius of Sgr $\mathrm{A}^{*}$ is $\sim 4 \times 10^{-4} \mathrm{mpc}$.

the mass of the pulsar can be neglected in comparison to the mass of the black hole, in general one PK parameter is sufficient to estimate the mass of $\mathrm{Sgr} \mathrm{A}^{*}$ with a precision at the $10^{-6}$ level. In the following we briefly discuss three relativistic effects that can be used for a mass determination, and present the results of extensive simulations at the end of this Section. As mentioned in Section [1, in a discussion of measurement precision it is sufficient to keep the first order terms in the description of these effects.

\subsection{Post-Keplerian parameters and mass determination}

In eccentric binary pulsars the precession of periastron, $\dot{\omega}$, is usually the first PK parameter that can be measured with high precision. It allows the determination of the total mass of the system, which in our case can be equaled with the mass of the black hole $M_{\mathrm{BH}}$. To first order one finds (Robertson 1938):

$$
\begin{aligned}
\dot{\omega} & \simeq \frac{3}{1-e^{2}}\left(\frac{2 \pi}{P_{\mathrm{b}}}\right)^{5 / 3}\left(\frac{G M_{\mathrm{BH}}}{c^{3}}\right)^{2 / 3} \\
& \simeq\left(0.269^{\circ} / \mathrm{yr}\right) \frac{1}{1-e^{2}}\left(\frac{P_{\mathrm{b}}}{1 \mathrm{yr}}\right)^{-5 / 3}\left(\frac{M_{\mathrm{BH}}}{4 \times 10^{6} M_{\odot}}\right)^{2 / 3},
\end{aligned}
$$

where $e$ is the orbital eccentricity. As an example, for a 0.3 yr orbit with an eccentricity of 0.5 the orbit precesses with a rate of about 2.7 degrees per year. After five years of weekly observations with a timing uncertainty of $100 \mu \mathrm{s}$, this precession will be measured with a fractional precision of better than $10^{-7}$. This, however, is not the precision with which the mass of Sgr $\mathrm{A}^{*}$ can be determined. If the black hole is rotating, a significant fraction of the pericenter precession can come from the frame dragging (Barker \& O'Connell 1975). Depending on the orientation and the spin of the black hole, this could be up to about $1 \%$ of the total precession. A mea- 
surement of the spin and the orientation of the black hole would allow to correct for this Lense-Thirring contribution $\dot{\omega}_{\mathrm{LT}}$. But as we will show later, we will use the observed $\dot{\omega}$ and the mass measurement from other relativistic effects to calculate $\dot{\omega}_{\mathrm{LT}}$ and use it in the spin determination $\left(\dot{\omega} \equiv \dot{\omega}_{\mathrm{M}}+\dot{\omega}_{\mathrm{LT}}\right)$.

The Einstein delay is a combination of the second order Doppler effect and gravitational redshift. From its amplitude $\gamma_{\mathrm{E}}$, which is a PK parameter, one can determine the mass of the black hole, since (to first post-Newtonian order) (Blandford \& Teukolsky 1976)

$$
\begin{aligned}
\gamma_{\mathrm{E}} & \simeq 2 e\left(\frac{P_{\mathrm{b}}}{2 \pi}\right)^{1 / 3}\left(\frac{G M_{\mathrm{BH}}}{c^{3}}\right)^{2 / 3} \\
& \simeq(2500 \mathrm{~s}) e\left(\frac{P_{\mathrm{b}}}{1 \mathrm{yr}}\right)^{1 / 3}\left(\frac{M_{\mathrm{BH}}}{4 \times 10^{6} M_{\odot}}\right)^{2 / 3} .
\end{aligned}
$$

For a 0.3 yr orbit with an eccentricity of 0.5 the amplitude of the Einstein delay will be of order 800 seconds. However, the Einstein delay is a priori not separable from the Roemer delay 6 , and can only be measured with sufficient accuracy after some time, when the relativistic precession has changed the orbital orientation sufficiently. For a pulsar in an $0.3 \mathrm{yr}$ orbit this is already the case after a few orbits. After a few years the Einstein delay can be measured with high precision, as will be shown in the simulations below. The dragging of inertial frames in the vicinity of the black hole also affects the Einstein delay. However, this occurs only at higher orders (Wex 1995), which in most cases can be neglected or easily accounted for in a combined mass and spin measurement.

The Shapiro delay accounts for the extra light travelling time due to the curvature of space-time caused by the existence of surrounding masses (here mainly Sgr A*). The Shapiro delay contains two separately measurable PK parameters, the mass of the black hole $M_{\mathrm{BH}}$ and $\sin i$. The signal is usually only sufficiently strong for edge-on systems (e.g. Kramer et al. 2006), but in our case even for a face-on orbit $(i=0)$ the effect will be significant due to the large mass of Sgr $\mathrm{A}^{*}$, if the orbit is eccentric. Using the equation of Blandford \& Teukolsky (1976),

$$
\begin{aligned}
\Delta_{\mathrm{S}} & \simeq \frac{2 G M_{\mathrm{BH}}}{c^{3}} \ln \left(\frac{1+e \cos \varphi}{1-\sin i \sin (\omega+\varphi)}\right) \\
& \simeq(39.4 \mathrm{~s})\left(\frac{M_{\mathrm{BH}}}{4 \times 10^{6} M_{\odot}}\right) \ln \left(\frac{1+e \cos \varphi}{1-\sin i \sin (\omega+\varphi)}\right),
\end{aligned}
$$

as a first order estimation, one can see that for an eccentricity of 0.5 the Shapiro delay for $i=0$ amounts to about 40 seconds. This already indicates that the Shapiro delay allows a precise mass determination, even for a pulsar with poor timing precision. Apart from containing $M_{\mathrm{BH}}$ directly, the Shapiro delay gives a second, though indirect, access to the $\operatorname{Sgr} \mathrm{A}^{*}$ mass via $\sin i$ and

\footnotetext{
${ }^{6}$ The Roemer delay is defined as $\Delta_{\mathrm{R}}=-\hat{\mathbf{K}}_{0} \cdot \mathbf{r}$, where $\hat{\mathbf{K}}_{0}$ is the unit vector of the line-of-sight and $\mathbf{r}$ is the position vector of the pulsar with respect to the barycenter of the binary system. The Roemer delay describes the contribution of the pulsar motion to the time delay.
}

the mass function. One finds

$$
G M_{\mathrm{BH}} \simeq\left(\frac{c x}{\sin i}\right)^{3}\left(\frac{2 \pi}{P_{\mathrm{b}}}\right)^{2},
$$

where $x$ is the projected semi-major axis of the pulsar orbit (in light seconds), which is an observable Keplerian parameter. It depends on the orbital eccentricity and inclination, which of the two is more constraining.

In addition, there are significant contributions to the signal propagation caused by frame dragging. A first order analytic equation for this effect can be found in Wex \& Kopeikin (1999). From this it is clear that the frame dragging can have a significant contribution to the propagation delay, but in most cases will have a distinct signature that can be fitted for, leading at the same time to a precise mass measurement and a lower limit on the spin parameter $\chi$. Contributions from higher order multipole moments and light bending effects can easily be accounted for in an analytic way (see e.g. Kopeikin 1997).

The inclination of the pulsar orbit with respect to the line-of-sight $i$ (modulo a $\pi-i$ ambiguity, see Fig. 4) can be obtained either directly from the Shapiro delay, as explained above, or via Eq. (8) by using the mass, $M_{\mathrm{BH}}$, derived from any other PK parameter. Therefore, in Section 4 and 5] where the determination of spin and quadrupole is presented, we can treat the inclination angle as a parameter that is known with sufficient precision. A brief discussion on the $\pi-i$ ambiguity can be found in Section 4.1

\subsection{Simulations}

The simulations performed in this paper mainly contain two steps: creating TOAs and determining parameters as well as their measurement uncertainties. Firstly, the TOAs are created regularly regarding to solar system barycentric time and then combined with the three time delays (Roemer, Einstein and Shapiro, see the above Subsection) to account for the changes in the signal arrival time due to the orbital motion of the pulsar around Sgr A*. Next the simulated TOAs are passed to the TEMPO software package. Based on a timing model, TEMPO performs a least-square fit to yield a phaseconnected solution of the TOAs, and determines the model parameters. The measurement uncertainties of these parameters are calculated via a covariance matrix. This is the standard procedure of pulsar timing observations, and explained in great detail in Tavlor (1994); Lorimer \& Kramer (2005); Hobbs et al. (2006); Edwards et al. (2006). Most of the timing models used in this paper are part of the TEMPO standard implementation available as a download from the sources given in these references. When ever we use an extension to these well tested models, to account for specific effects, which are not covered by the standard software, we will mention this explicitly in the corresponding Section.

In this Subsection we present the simulations for the mass determination. For this we assumed five years of observations with weekly TOAs which contain white Gaussian noise with a standard deviation of $100 \mu \mathrm{s}$. Fig. 3 shows the results of our simulations for a typical system configuration. If this is not the case then, as outlined above, $\dot{\omega}$ cannot a priori be used for a high precision mass measurement due to an unknown contribution from the 
frame dragging, as we will show later.

In practice, not only one single relativistic effect will be used to determine the mass of Sgr A*, but a consistent model, accounting simultaneously for frame dragging effects in the orbital motion and the signal propagation, will be used to determine the mass and spin at the best level. How the spin of Sgr A* affects the timing observations and how it can be extracted from the timing data is the subject of the next Section.

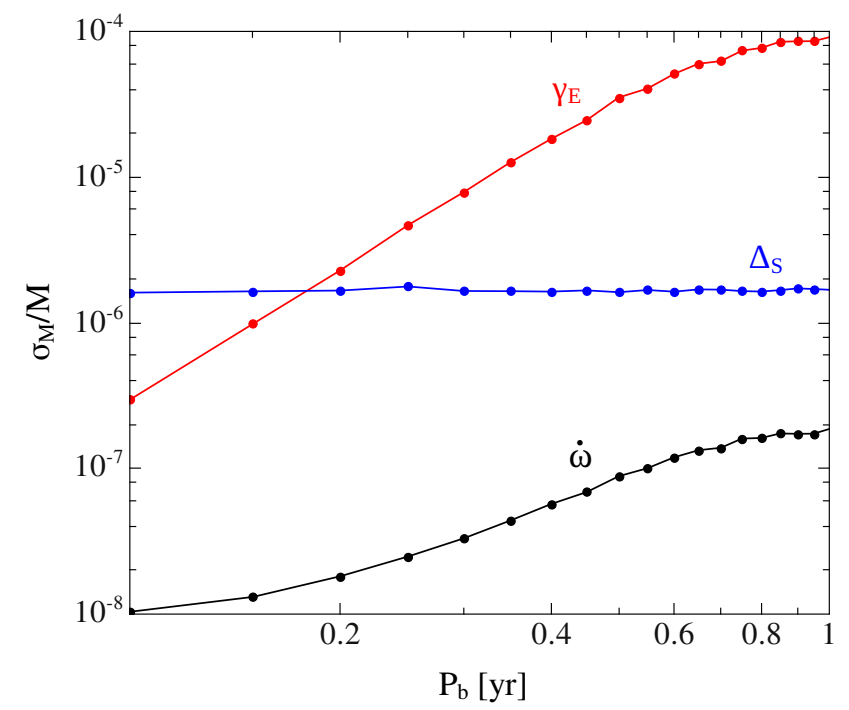

FIG. 3.- Simulated fractional precision for the mass determination of Sgr A* as a function of the orbital period $P_{\mathrm{b}}$, obtained from three different relativistic effects: precession of the orbit $(\dot{\omega})$, Einstein delay $\left(\gamma_{\mathrm{E}}\right)$, and Shapiro delay $\left(\Delta_{\mathrm{S}}\right)$. The mass determinations are based on simulated data, assuming weekly TOAs with an uncertainty of $100 \mu$ s over a time span of five years. We used an orbital eccentricity $e$ of 0.5 and an orbital inclination $i$, relevant for the Shapiro delay, of $60^{\circ}$. The simulations were done for a nonrotating black hole. Note that for various practical reasons (such as the uncertainty in the pulsar mass), a precision below $10^{-7}$ seems unrealistic. Also, as explained in the text, for a rotating black hole $\dot{\omega}$ cannot be used directly for a high-precision mass determination, due to the large contribution of frame dragging.

\section{FRAME DRAGGING, SPIN MEASUREMENT, AND GR'S COSMIC CENSORSHIP CONJECTURE}

Although there are clear indication that the Sgr A* rotates, its actual rate of rotation is still not well determined. Investigations of flares from accreting gas in the near-infrared and in X-rays yield a range of $\chi \approx$ 0.22 to 0.99 (Genzel et al. 2003; Aschenbach et al. 2004; Bélanger et al. 2006; Aschenbach 2010). The rather large range in the estimates of $\chi$ is also a result of the uncertainty in the underlying model assumptions. A pulsar, however, would provide a clean probe of the gravitational field of Sgr A* and, in absence of any major external perturbations, give a direct access to the dragging of inertial frames in the vicinity of Sgr A*. In Wex \& Kopeikin (1999) it has been shown that in relativistic pulsar-black hole binaries the (additional) precession of a pulsar orbit due to the frame dragging caused by the spin of the black hole (Lense-Thirring precession) is the most promising effect to determine the direction and magnitude of the black hole spin. This, in general, is also the case for a pulsar in orbit about Sgr A*. The assumption made in Wex \& Kopeikin (1999), that the spin of the black hole $S$ is clearly smaller than the orbital angular momentum $L$, is no longer valid here. The fraction between the spin of the black hole and the angular orbital momentum is given by

$$
\frac{S_{\mathrm{BH}}}{L}=\frac{M_{\mathrm{BH}}}{M_{\mathrm{PSR}}} \beta_{e} \chi,
$$

where $\beta_{e}=\beta_{\mathrm{O}} / \sqrt{1-e^{2}}$ and $M_{\mathrm{PSR}}$ is the mass of the pulsar. For a pulsar with an orbital period of less than one year $S_{\mathrm{BH}} / L$ is greater than $50000 \chi$. Thus, the total angular momentum $\mathbf{J}$ of the system is completely dominated by the spin of Sgr A*, whose direction will therefore practically coincide with the direction of $\mathbf{J}$, and can, for the considerations here, be viewed as a constant in time. In this case, the orbital motion to post-Newtonian accuracy including first-order spin terms can be taken from Appendix B in Wex (1995). This is sufficient to simulate all the relevant effects (see Königsdörffer \& Gopakumar 2005, for higher order corrections) for a system free of external perturbations. It also accounts for the fact that the precession is stronger near the pericenter.

\subsection{Spin determination from the timing parameters}

Averaging over one orbit, one obtains the rates of the secular precession of the pulsar orbit caused by frame dragging (Barker \& O'Connell 1975)]:

$$
\left.\begin{array}{l}
\dot{\Phi}=\Omega_{\mathrm{LT}} \\
\dot{\Psi}=-3 \Omega_{\mathrm{LT}} \cos \theta
\end{array}\right\} \quad \Omega_{\mathrm{LT}}=\frac{4 \pi}{P_{\mathrm{b}}} \beta_{e}^{3} \chi \equiv \hat{\Omega} \chi .
$$

The definitions of the angles $\Phi, \Psi$ and $\theta$ are given in Fig. 4. The secular changes for the angles $\Phi$ and $\Psi$ are linear in time. As discussed in detail in Wex (1998) and Wex \& Kopeikin (1999), this linear-in-time evolution translates into a non-linear-in-time evolution of the observable angles that enter the timing model for a pulsar, i.e. the longitude of pericenter $\omega$ and the inclination of the orbit with respect to the line-of-sight $i$ (as part of the projected semi-major axis $x$ ). One finds 8

$$
c_{i}=c_{\theta} c_{\lambda}-s_{\theta} s_{\lambda} c_{\Phi}
$$

and

$$
\sin (\omega-\Psi)=\frac{s_{\lambda} s_{\Phi}}{s_{i}}, \quad \cos (\omega-\Psi)=\frac{c_{\lambda}-c_{\theta} c_{i}}{s_{\theta} s_{i}} .
$$

Since the angles $i, \theta$, and $\lambda$ are in the range 0 to $\pi$, their sines $s_{X}$ are non-negative and can be expressed as $s_{X}=\sqrt{1-c_{X}^{2}}$. As shown by Wex $(1998)$, if the change in $\Phi$ is small (less than a few degrees) over the time span of the timing observations, the most straightforward way to analyze the timing data is to fit for the coefficients of the Taylor expansion of $\omega(t)$ and $x(t)$

$$
\begin{aligned}
& \omega=\omega_{0}+\dot{\omega}_{0}\left(t-t_{0}\right)+\frac{1}{2} \ddot{\omega}_{0}\left(t-t_{0}\right)^{2}+\ldots, \\
& x=x_{0}+\dot{x}_{0}\left(t-t_{0}\right)+\frac{1}{2} \ddot{x}_{0}\left(t-t_{0}\right)^{2}+\ldots,
\end{aligned}
$$

7 To estimate the measurability of the Lense-Thirring effect, it is sufficient to use the averaged precession rate. In practice, the preccession of the orbital plane is more complicated as can be seen from the analytic solution given in Appendix B of Wex (1995)).

${ }^{8}$ We define $c_{X} \equiv \cos X$ and $s_{X} \equiv \sin X$. 
and to use the parameters $\omega_{0}, x_{0}$ and their time derivatives as intermediate parameters to determine the angles $\theta, \lambda, \Phi_{0}, \Psi_{0}$, and the spin parameter $\chi$. For the configurations considered in this paper it is sufficient to keep only terms up to second order in $t-t_{0}$. Nevertheless, we have extended TEMPO to account for cubic terms in oder to test their significance in all our simulations. We would like to note in passing, that the coefficients of the cubic terms can be calculated from the other coefficients based on basic geometric relations, and therefore they would not add further information concerning the orientation of the system and the spin magnitude.

From the derivatives of Eqs. (11) and (12) one finds the relation between the time derivatives, the orientation of the orbit at a given epoch, and the spin of Sgr A*. In practice, the linear trend becomes visible in the timing data soon after the beginning time of observation, allowing the measurement of $\dot{x}_{0}$ and the extraction of the Lense-Thirring contribution from $\dot{\omega}_{0}$. One finds (for convenience we drop the index 0 ):

$$
\begin{aligned}
\dot{x} & =-x s_{i}^{-2} c_{i} s_{3} \Omega_{\mathrm{LT}}, \\
\dot{\omega}-\dot{\omega}_{M} & =s_{i}^{-2}\left[\left(1-3 s_{i}^{2}\right) c_{\theta}-c_{i} c_{\lambda}\right] \Omega_{\mathrm{LT}},
\end{aligned}
$$

where $s_{3} \equiv s_{\theta} s_{\lambda} s_{\Phi}$. Since at this point $x,\left|c_{i}\right|$, and $s_{i}$ are known quantities, the measurement of $\dot{x}$ determines the quantity $\left|s_{3}\right| \chi$ which must not exceed unity, since according to the CCC $\chi \leq 1$ and $\left|s_{3}\right| \leq 1$ by definition. Hence, as soon as $\dot{x}$ becomes measurable one has a first test for the CCC.

To fully determine the magnitude and orientation of the spin, the measurement of higher order derivatives is necessary. The second time derivatives read

$$
\begin{aligned}
& \ddot{x}=-x s_{i}^{-4}\left[s_{3}^{2}+s_{i}^{2} c_{i}\left(c_{\theta} c_{\lambda}-c_{i}\right)\right] \Omega_{\mathrm{LT}}^{2}, \\
& \ddot{\omega}=s_{i}^{-4}\left[2 c_{i} c_{\theta}-\left(2-s_{i}^{2}\right) c_{\lambda}\right] s_{3} \Omega_{\mathrm{LT}}^{2},
\end{aligned}
$$

which give us now, in total, six equations (11, 12, 15, 16. 17, 18) for five unknowns $\left(\theta, \lambda, \Phi_{0}, \Psi_{0}, \chi\right)$. For a discussion of the solution of these equations, we introduce the variables $\chi_{\theta} \equiv c_{\theta} \chi, \chi_{\lambda} \equiv c_{\lambda} \chi$, and $\zeta_{3} \equiv s_{3} \chi$. The parameters $\chi_{\theta}$ and $\chi_{\lambda}$ represent the projection of the (normalized) spin onto the orbital angular momentum and the line-of-sight direction, respectively. They can be determined from the timing parameters via the equations of the first time derivatives

$$
\begin{gathered}
-\frac{\dot{x} s_{i}^{2}}{x \hat{\Omega}} \equiv \mathcal{X}_{1}=c_{i} \zeta_{3}, \\
\frac{\left(\dot{\omega}-\dot{\omega}_{M}\right) s_{i}^{2}}{\hat{\Omega}} \equiv \mathcal{W}_{1}=\left(1-3 s_{i}^{2}\right) \chi_{\theta}-c_{i} \chi_{\lambda},
\end{gathered}
$$

and those of the second time derivatives

$$
\begin{aligned}
\frac{\left(\ddot{x} x c_{i}^{2}+\dot{x}^{2} s_{i}^{2}\right) s_{i}^{4}}{\left(x \hat{\Omega} c_{i}\right)^{2}} & \equiv \mathcal{X}_{2}=c_{i}^{2}\left(\chi_{\theta}^{2}+\chi_{\lambda}^{2}\right)-c_{i}\left(1+c_{i}^{2}\right) \chi_{\theta} \chi_{\lambda}, \\
-\frac{\ddot{\omega} x c_{i}^{2} s_{i}^{2}}{\dot{x} \hat{\Omega}} & \equiv \mathcal{W}_{2}=2 c_{i}^{2} \chi_{\theta}-c_{i}\left(1+c_{i}^{2}\right) \chi_{\lambda} .
\end{aligned}
$$

where $\zeta_{3}$ has been eliminated using Eq. (19). The quantities $\mathcal{X}_{1}, \mathcal{W}_{1}, \mathcal{X}_{2}$, and $\mathcal{W}_{2}$ are defined such that they do not change when the sign of $c_{i}$ is flipped. The above equations can be easily solved analytically. By the time the second derivatives are observable, $s_{i}, \dot{x}, \dot{\omega}_{\mathrm{LT}}$, and so $\mathcal{X}_{1}$ and $\mathcal{W}_{1}$, will be known with high precision. For a given sign of $c_{i}= \pm \sqrt{1-s_{i}^{2}}$, Eq. (22) will lead to a unique solution for $\chi_{\theta}, \chi_{\lambda}$, and $\zeta_{3}$. For some orientations Eq. (21) turns out to be more constraining. However, this gives us in general two solutions for $\left(\chi_{\theta}, \chi_{\lambda}\right)$. But then Eq. (22), although less constraining, can be used to rule out one of the two solutions. The best way to represent the solution is to plot the constraints from Eqs. (20), (21) and (22) in the $\chi_{\theta}-\chi_{\lambda}$ plane. Possible solutions are represented by the region where all three curves meet within the uncertainty given by the measurement errors of $\dot{x}, \dot{\omega}_{\mathrm{LT}}, \ddot{x}$, and $\ddot{\omega}$. This will become clear in the Section below, where we present the simulations.

With $\chi_{\theta}, \chi_{\lambda}$ and $\zeta_{3}$ known, we can calculate the spin parameter of the black hole via

$$
\chi=s_{i}^{-1} \sqrt{\zeta_{3}^{2}+\chi_{\theta}^{2}+\chi_{\lambda}^{2}-2 c_{i} \chi_{\theta} \chi_{\lambda}} .
$$

Once $\chi$ is determined, we can calculate all the angles. Finally, the $i \leftrightarrow \pi-i$ ambiguity leaves us with two different solutions in the orientation by $\left(\Phi_{0}, \lambda\right) \leftrightarrow\left(\pi+\Phi_{0}, \pi-\lambda\right)$, for which $\chi$ has the same value.

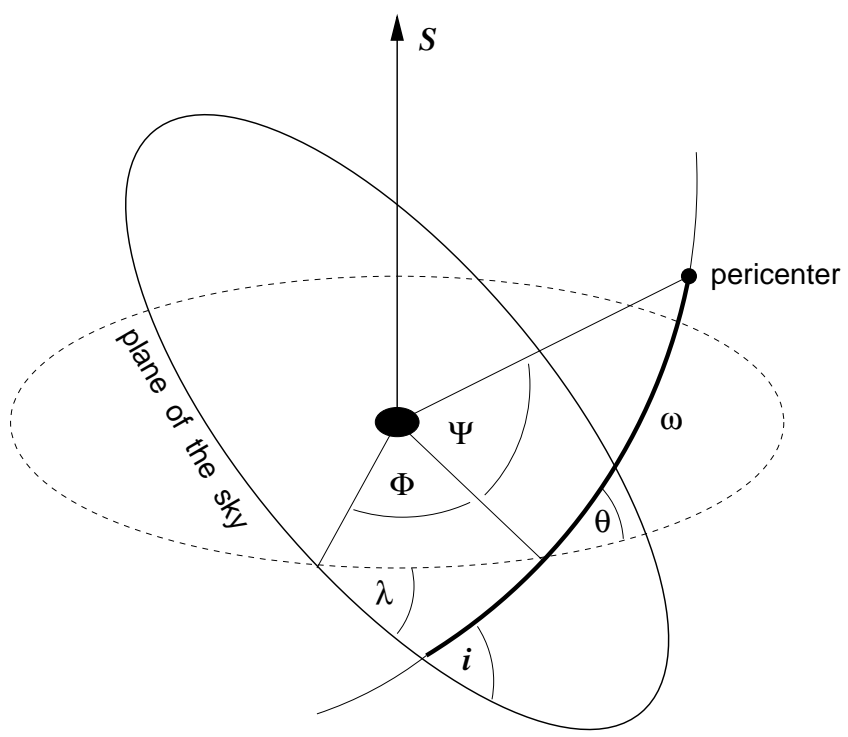

FIG. 4.- Definition of angles in Sgr A* spin reference frame. The orientation of the orbit with respect to the observer is given by the orbital inclination $i$ and the longitude of pericenter $\omega$ as measured from the ascending node in the plane of the sky. The pulsar orbit with respect to the equatorial plane of the rotating black hole is determined by the inclination $\theta$, the equatorial longitude of the ascending node $\Phi$, and the equatorial longitude of pericenter $\Psi$. The angle between the line-of-sight and the Sgr A* spin is denoted by $\lambda$.

\subsection{Simulations}

The technique described in the previous Subsection has been tested by a set of standard simulations for various orbital configurations. For a given system, following the procedures described in Section 3.2 we simulate weekly $100 \mu \mathrm{s}$ TOAs over a time span of five years. Here in the calculations of the time delays, in addition to the relativistic pericenter advance we also consider the influence of $\operatorname{Sgr} \mathrm{A}^{*}$ spin by inputting the secular changes of $\Phi$ and 
$\Psi$ described in Eq. (10). Then, in order to determine the PK parameters, we fit the TOAs with the MSS timing model of TEMPO, which we have extended to model the secular changes in pericenter and projected semi-major axis up to the third order in the time derivatives. The third order coefficient turn out not to be significant in the simulations presented here. Figs. 5 and 6 show the $\chi_{\theta}{ }^{-} \chi_{\lambda}$ plane for two different orientations of the black hole and the pulsar orbit. According to GR the solution has to lie within the boundaries of the figures, since $-1 \leq \chi_{\theta}, \chi_{\lambda} \leq 1$ for a Kerr black hole. Moreover, the solution $\left(\chi_{\theta}, \chi_{\lambda}\right)$ has to lie within an ellipse defined by setting $\chi=1$ in Eq. (23), in order to represent a Kerr black hole with an event horizon. Once $\dot{x}$ is measured, one can determine $\zeta_{3}$ from Eq. (19) and use this quantity to plot the ellipse defined by Eq. (23) in the $\chi_{\theta}-\chi_{\lambda}$ plane.

Fig. 7 shows a simulation for a Kerr solution with a spin that exceeds the spin of an extreme Kerr black hole. Within GR, this would represent a naked singularity. For such an object the CCC is violated and the predictability of the (classical) theory breaks down. Also, all three lines have to agree in a common region, otherwise either GR is not the correct theory, or there are external perturbations present, a situation which we discuss in more details in the next Section.

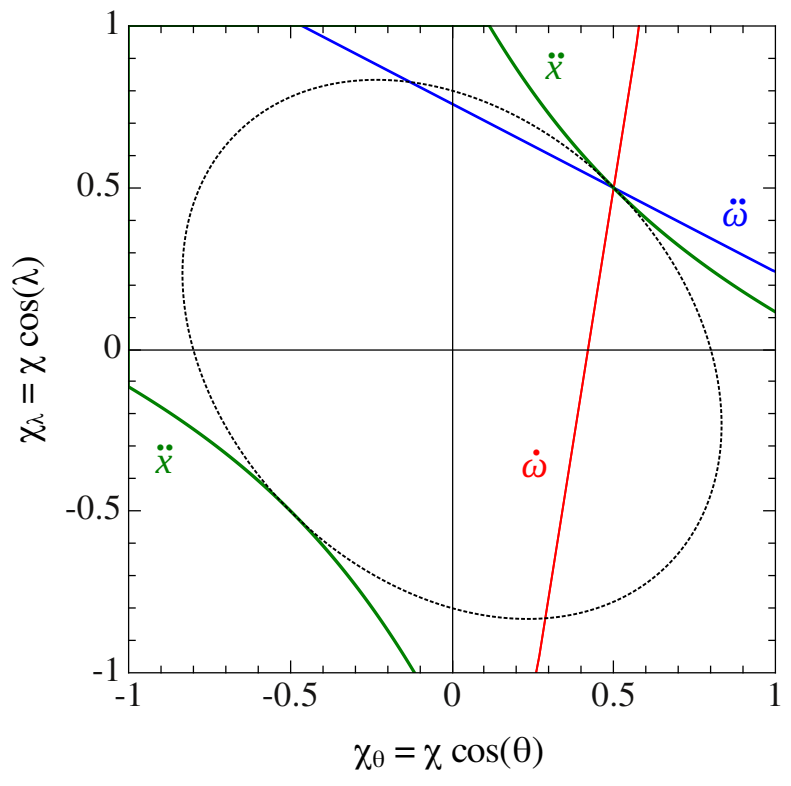

FIG. 5.- Determination of the Sgr A* orientation in the $\chi_{\theta^{-}}$ $\chi_{\lambda}$ plane. For this simulation we have used an orbital period of $0.3 \mathrm{yr}$, an orbital eccentricity of $0.5, \chi=1, \Psi_{0}=45^{\circ}, \Phi_{0}=45^{\circ}$, $\theta=60^{\circ}$, and $\lambda=60^{\circ}$, which are in agreement with the constraints by Zamaninasab et al. (2011). A change in the sign of $c_{i}$ mirrors the figure along the $\chi_{\lambda}=0$ line, meaning that the solution for $\theta$ is invariant, but $\lambda$ changes to $\pi-\lambda$. The corresponding spin parameter, as calculated from Eq. (23), is $\chi=0.9997 \pm 0.0010(95 \%$ C.L.). In all the $\chi_{\theta}-\chi_{\lambda}$ plots (Fig. [5]- we plot the $68 \%$ confidence intervals. However, in most cases the separation between the two lines is below the resolution of the plot. The dotted ellipse is the boundary of the area for Kerr black holes (see text for details).

\subsection{Identification of external perturbations}

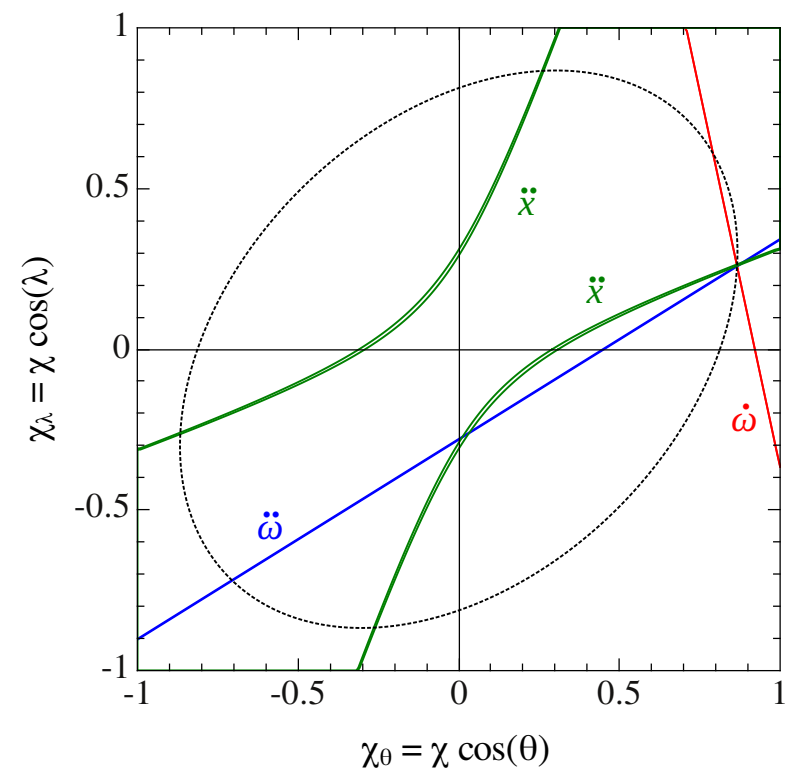

Fig. 6. - Like Fig. 5 but $\Phi_{0}=105^{\circ}, \theta=30^{\circ}$, and $\lambda=75^{\circ}$. The corresponding spin parameter, as calculated from Eq. (23), is $\chi=1.0001 \pm 0.0003$ (95\% C.L.).

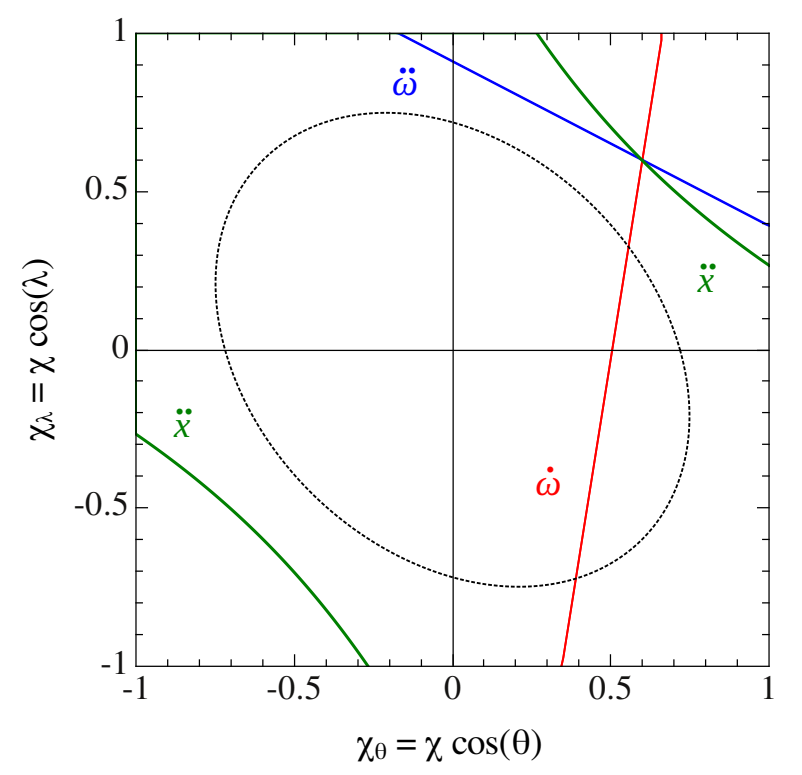

Fig. 7.- Parameters as in Fig. 5 but $\chi=1.2$ (naked Kerr singularity). The dotted ellipse is the (outer) border of the region where, for the measured orbital inclination and $\dot{x}$, the Kerr black holes are located, i.e. where $\chi \leq 1$.

As discussed in great detail by Merritt et al. (2010), the orbit of a star or pulsar around $\mathrm{Sgr} \mathrm{A}^{*}$ may be subject to perturbations from other stars in the vicinity of the black hole. Depending on the number density of the stars, this could significantly affect the precession of the pulsar orbit. Nevertheless, since we have three lines in the $\chi_{\theta}-\chi_{\lambda}$ plane that need to intersect, our analysis will unveil the presence of any external perturbations. In Fig. 8 we present a $\chi_{\theta}-\chi_{\lambda}$ diagram based on timing data 
that contain (besides the gravitational field of $\operatorname{Sgr} \mathrm{A}^{*}$ ) an external perturbation causing an additional precession of the pericenter. For orbits with $P_{\mathrm{b}} \lesssim 0.3 \mathrm{yr}$, even a small (compared to the Lense-Thirring precession) external contribution to the precession of the pericenter leads to a situation where the $\dot{\omega}, \ddot{\omega}$, and $\ddot{x}$ lines fail to intersect in one point within the measurement precision. The same is true, if there is an external contribution to a change in the inclination of the orbital plane. Hence, if all three lines intersect, we not only have a precise determination of the spin of the black hole, but also a test that this measurement is not contaminated by external perturbations.

At this point we would like to add a more detailed comment on the discriminating power of the pulsar test concerning external perturbations. In practice, the threeline test outlined above is not simply based on the secular precession rates. We emphasize that a consistent fit of the timing data, with a model that includes the Lense-Thirring precession, needs to incorporate the full dynamics of the orbital precession as given by Appendix $\mathrm{B}$ in Wex (1995). The phase dependence of the LenseThirring precession rate is a direct result of the Coriolis type force caused by the dipolar gravitomagnetic field of the central rotating black hole. Hence, we can identify an external perturbation based on this quasi-periodic effect, even in a fine tuned situation where the external mass distribution manages to mimic a secular Lense-Thirring precession. In fact, we have conducted simulations and found that the phase dependent precession rate leads to an effect that is typically four orders of magnitude larger than the timing precision assumed in our simulations. This is in line with the findings of Damour \& Deruelle (1986), who pointed out the strength of quasi-periodic effects in tests of gravity.

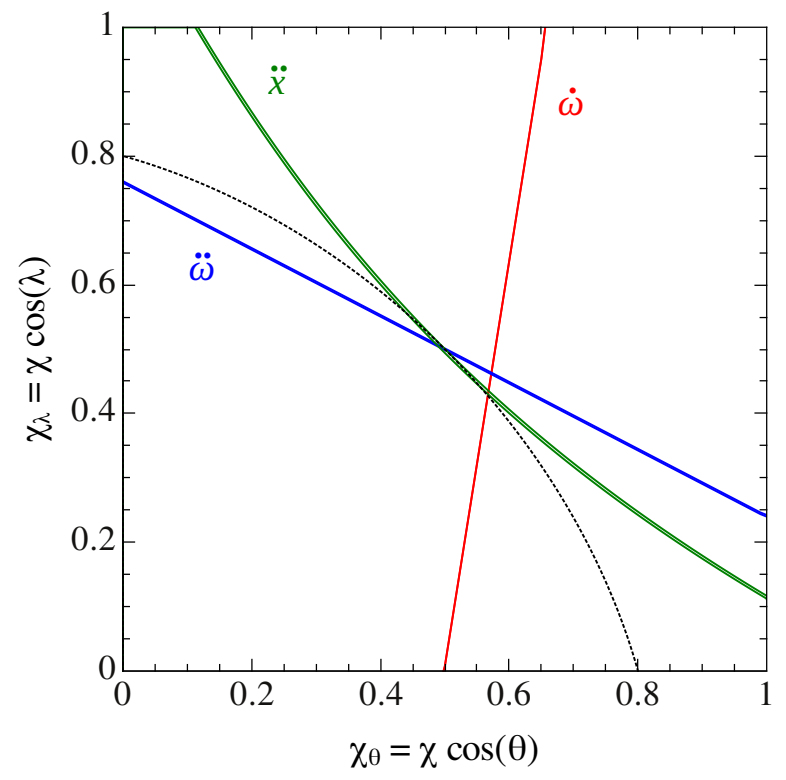

Fig. 8.- Parameters as in Fig. 5 but the precession of pericenter has an additional contribution from an external perturbation that amounts to $10 \%$ of the Lense-Thirring contribution. For a better resolution only the first quadrant of Fig. 5 is plotted here.

\section{QUADRUPOLE MEASUREMENT AND NO-HAIR THEOREM TEST}

The quadrupole moment of the black hole leads to an additional secular precession of the pulsar orbit. This precession, however, is even for compact orbits $\left(P_{b} \sim\right.$ $0.1 \mathrm{yr})$ much smaller than the Lense-Thirring precession. Further, it can be shown that the secular terms of the precession cannot be separated from the LenseThirring effect. For this reason, it has been argued by Wex \& Kopeikin (1999) that while the spin magnitude and the orientation of the black hole are mainly determined by the overall precession of the orbit, the quadrupole of the black hole is mostly determined via its periodic influence on the motion of the pulsar from one pericenter to the next. As will be shown in this Section, these periodic features of the quadrupole can be used to fit for the quadrupole moment of Sgr A*.

\subsection{Extracting the quadrupole from the timing data}

The deviations in the motion of the pulsar caused by the quadrupole moment lead to a variation in the Roemer delay, which we describe by a change in the coordinate position of the pulsar according to

$$
\mathbf{r}^{\prime}=\left(r+\delta r^{(q)}\right)\left(\hat{\mathbf{n}}+\delta \hat{\mathbf{n}}^{(q)}\right) .
$$

The vector $\delta \hat{\mathbf{n}}$ is calculated from the changes in the angles

$$
\Phi^{\prime}=\Phi+\delta \Phi^{(q)}, \quad \Psi^{\prime}=\Phi+\delta \Psi^{(q)}, \quad \theta^{\prime}=\theta+\delta \theta^{(q)},
$$

according to $\delta \hat{\mathbf{n}}=\hat{\mathbf{n}}^{\prime}-\hat{\mathbf{n}}$. To first order in $\epsilon \equiv$ $-3 Q / a^{2}\left(1-e^{2}\right)^{2}$, the detailed equations for the $\delta$ quantities can be taken from Garfinkel (1959), with slight modifications that account for the dominating precession of pericenter caused by the mass monopole: the term $\left(5 y^{2}-1\right)$ in the auxiliary constants $m$ and $\gamma$ has to be replaced by $2 \dot{\omega} P_{\mathrm{b}} / \pi \epsilon$, where $\dot{\omega}$ is the total advance of pericenter. Based on this we have developed a timing model that includes the contribution of the quadrupole moment of $\mathrm{Sgr}^{*}$ to first order in $\epsilon$. Fig. 9 illustrates the unique periodic timing residuals caused by the quadrupole moment of Sgr A*.

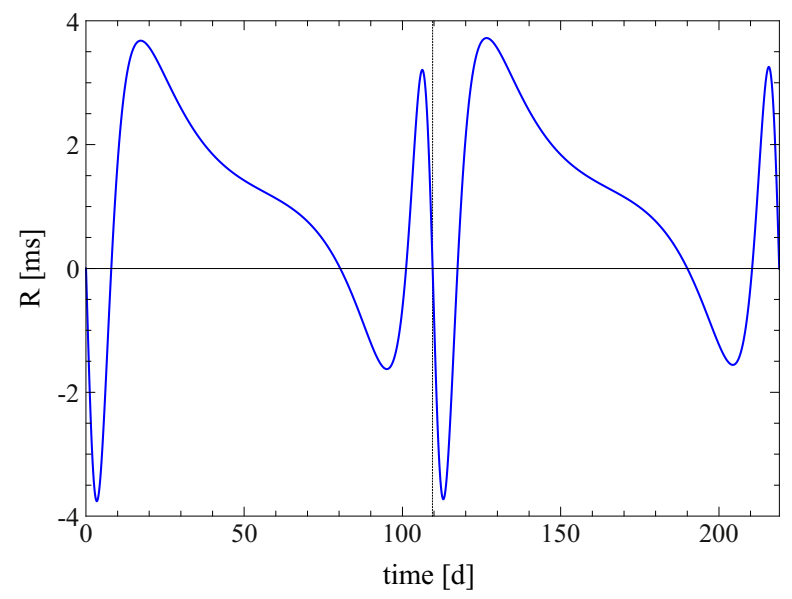

Fig. 9.- Residuals caused by the quadrupole moment of Sgr A* plotted for two orbital phases. We have used the same orbital and black hole parameters as in Fig. [5] 
This periodic signal will not only allow the determination of the quadrupole moment of Sgr A* with high precision, but also provide a clear identification of the quadrupolar nature of the gravitational field. Moreover, due to the large advance of pericenter the quadrupolar signal will change in a characteristic way from one orbit to the next. This clearly helps to identify any external "contamination" of the orbital motion of the pulsar, and, as in the spin determination, provides high confidence in the reliability of a no-hair theorem test with a pulsar around Sgr A*.

\subsection{Simulations}

We have tested the procedure outlined above in a number of mock data simulations, for various orbital configurations. Again following the procedures described in Section 3.2 we assume weekly TOAs with a precision of $100 \mu \mathrm{s}$ for a time span of five years. This time we extended our simulations and the timing model used in Section 4.2 to account for the periodic effects due to quadrupole moment of Sgr A* described in Eq. (24). Our results are summarized in Fig. 10, Note that the precision of the spin determination is at least one order of magnitude better than the determination of $q$. Hence the uncertainty in the $q$-measurement is the limiting factor for the no-hair theorem test. As a conclusion of our simulations, if the external perturbations are negligible, for orbits with $P_{\mathrm{b}} \lesssim 0.5 \mathrm{yr}$ the no-hair theorem can be tested with high precision. If we adopt the precessional rates from stellar perturbation calculated in Fig. 2, we conclude that the test can be achieved with high precision for orbits with $P_{\mathrm{b}} \lesssim 0.1 \mathrm{yr}$. This range can be extended if in the presence of perturbations the characteristic quadrupolar features remain separable. This, however depends on the details of the external mass distribution, which we will not investigate further in this paper.

\section{DISCUSSION}

In this paper we have developed a method to determine the mass, the spin, and the quadrupole moment of Sgr A* using a pulsar in a compact orbit around this super-massive black hole. Our investigation is based on a consistent timing model, that includes all the relativistic and precessional effects that can be used to extract these parameters of Sgr A*. Based on simulated timing data for a pulsar in orbit around Sgr A*, we have shown in a consistent covariance analysis, that even with a moderate timing precision $(\sim 100 \mu \mathrm{s})$, one can expect to be able to determine the mass, the spin, and the quadrupole moment of Sgr A* with high precision, provided the orbital period is well below one year. As a result of our simulations, for a compact orbit (orbital period of a few months) one can expect to measure the spin with a precision of $10^{-3}$, or even better. We have shown how the method would allow the identification of an object whose frame-dragging exceeds that of an extreme Kerr black hole, and therefore would provide a test of the CCC. Furthermore, for such orbits the determination of the quadrupole moment of Sgr A* seems feasible at a few percent precision level or even better, depending on the size and orientation of the pulsar orbit and the spin of Sgr A*. In combination with the precise spin measurement from the Lense-Thirring effect, this yields a high

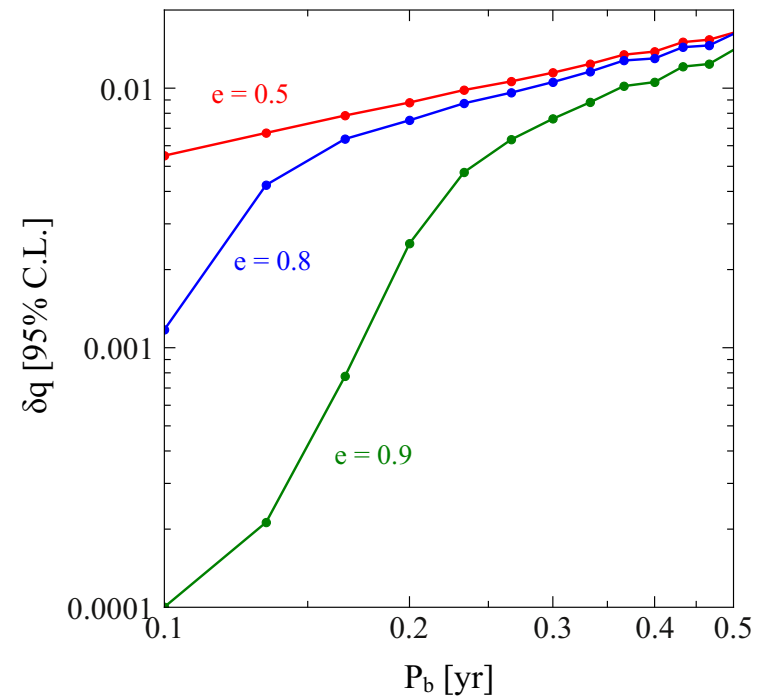

FIG. 10.- Measurement precision for the quadrupole moment of Sgr A* as a function of orbital period for three different eccentricities, in absence of any external perturbations. We have used the same orbital and black hole parameters as in Fig. 5 For the timing, we assumed the same time span and characteristics of TOAs as in Fig. 5] This time however the TOAs were equally distributed with respect to the true anomaly, in order to account for the fact that timing needs to be done more frequently around pericenter to optimize the measurement of the quadrupolar signal in the TOAs.

precision test of the no-hair theorem of stationary black holes.

Moreover, we have shown that, in general, our analysis will be able to unveil the presence of external perturbations caused by the presence of distributed mass, therefore providing high confidence in a spin and quadrupole determination based on pulsar timing. Nevertheless, further studies are required to see whether the spin and quadrupole moment can still be extracted if the timing data is "contaminated" by external perturbations. If perturbations arise from a smooth concentration of dark matter particles in the vicinity of Sgr A*, we may be able to learn something about the properties of dark matter that clusters around $\mathrm{Sgr} \mathrm{A}^{*}$, assuming GR is correct.

Finally, we need to emphasize that the tests presented are not affected by an uncertainty in the distance to the GC. On the contrary, a mass determination via pulsar timing would give a greatly improved value for $R_{0}$ if combined with the astrometric measurements in the near infrared.

Once a pulsar is detected in a compact orbit around Sgr A*, continuous timing will allow more and more measurements and tests as the timing baseline grows with time. In the following we summarize the most important steps in this experiment:

- After timing one orbit, all Keplerian parameters will be well known, and also the pericenter advance will be measured with good precision. This will already provide a good estimate of the mass of Sgr A*.

- Timing a few more orbits would then allow the determination of additional post-Keplerian parameters, like the Shapiro parameters $\left(r_{\mathrm{Sh}}, \sin i\right)$ and 
the amplitude of the Einstein delay $\left(\gamma_{\mathrm{E}}\right)$. These parameters allow a robust determination of Sgr A* mass and the inclination of the pulsar orbit with respect to the line-of-sight.

- At this stage, a measurement of a change in the projected semi-major axis $(\dot{x})$ caused by the LenseThirring will allow an early test of the CCC, and mapping of the region in the $\chi_{\theta}-\chi_{\lambda}$ plane where the solutions for Kerr black holes are.

- Around the same time the mass measurement should reach a precision that allows the extraction of the Lense-Thirring contribution to the precession of the pericenter $\left(\dot{\omega}_{\mathrm{LT}}\right)$, giving a line-like region in the $\chi_{\theta}-\chi_{\lambda}$ plane.

- After a few years of timing the second time derivatives of $\omega$ and $x$ should be known with high precision, allowing a precise determination of the Sgr A* spin (magnitude and direction). At this stage we also have a test for the "cleanness" of the system, and whether the spin is below the Kerr bound $(\chi=1)$.

- At the same time the obtained parameters for the pulsar orbit and the Sgr A* spin can be used to model the periodic features in the timing residuals, which are caused by the quadrupole moment of Sgr A*. This allows to a determination of the quadrupole moment and a test of the no-hair theorem.

A potential problem for the timing of a pulsar in a compact orbit around $\operatorname{Sgr~A}^{*}$ is posed by the relativistic spin precession, as pointed out by Merritt et al. (2010). This change in the pulsar orientation with respect to a distant observer not only causes a variation of the pulse profile, which makes precise timing more difficult, but also can turn the pulsar emission away from our line-of-sight (Weisberg et al. 1989; Kramer 1998). To leading order the spin-precession is given by the de Sitter precession rate, which for $M_{\mathrm{BH}} \gg M_{\mathrm{PSR}}$ reads (Barker \& O'Connell 1975)

$$
\Omega_{\mathrm{dS}} \simeq \frac{3 \pi}{P_{\mathrm{b}}} \beta_{e}^{2} \simeq\left(0.13^{\circ} / \mathrm{yr}\right) \frac{1}{1-e^{2}}\left(\frac{P_{\mathrm{b}}}{1 \mathrm{yr}}\right)^{-5 / 3} .
$$

\section{REFERENCES}

Angélil, R., Saha, P., \& Merritt, D. 2010, ApJ, 720, 1303 Aschenbach, B. 2010, Mem. Soc. Astron. Italiana, 81, 319 Aschenbach, B., Grosso, N., Porquet, D., \& Predehl, P. 2004, A\&A, 417, 71

Baganoff, F. K., et al. 2001, Nature, 413, 45

Barker, B. M., \& O'Connell, R. F. 1975, ApJ, 199, L25

Barker, B. M., \& O'Connell, R. F. 1975, Phys. Rev. D, 12, 329

Bélanger, G., Terrier, R., de Jager, O. C., Goldwurm, A., \&

Melia, F. 2006, Journal of Physics Conference Series, 54, 420

Blandford, R., \& Teukolsky, S. A. 1976, ApJ, 205, 580

Cordes, J. M., \& Lazio, J. T. W. 1997, ApJ, 475, 557

Cordes, J. M., \& Lazio, T. J. W. 2002, arXiv:astro-ph/0207156

Cordes, J. M., \& Shannon, R. M. 2010, astro-ph/1107.3086

Damour, T., \& Deruelle, N. 1986, Ann. Inst. H. Poincaré (Physique Théorique), 44, 263

Damour, T., \& Schäfer, G. 1988, Nuovo Cim., 101, 127

Damour, T., \& Taylor, J. H. 1992, Phys. Rev. D, 45, 1840

Deneva, J. S., Cordes, J. M., \& Lazio, T. J. W. 2009, ApJ, 702, L177
Consequently, for orbital periods below one year relativistic spin precession is expected to play an important role in the timing observations. We note in passing, that the Pugh-Schiff precession rate caused by frame dragging $\left(\Omega_{\mathrm{FD}} \sim 2 \pi \beta_{e}^{3} \chi / P_{\mathrm{b}}, \mathrm{Pugh} 1959\right.$; Schiff 1960$)$ is only relevant in the case of very compact, highly eccentric orbits $\left(\Omega_{\mathrm{FD}} \sim 1^{\circ} / \mathrm{yr}\right.$ for $P_{\mathrm{b}}=0.1 \mathrm{yr}, e=0.8$ and $\left.\chi=1\right)$, and could provide an independent test of the rotation of Sgr A*.

The no-hair theorem test can also be affected by the accretion disc around Sgr A*. To get an idea of the strength of this effect, one can estimate the influence by calculating the fraction of the quadrupolar potential of the disc to that of the black hole. This ratio is given by

$$
\mathscr{R} \sim M_{\mathrm{disc}} r_{\text {disc }}^{2} /\left(M_{\mathrm{BH}} r_{\mathrm{g}}^{2}\right),
$$

where $M_{\text {disc }}$ and $r_{\text {disc }}$ are the mass and outer radius of the disc, and $r_{\mathrm{g}} \equiv G M_{\mathrm{BH}} / c^{2}$ is the black hole gravitational radius. Following the advection-dominated accretion flow (ADAF) model of Yuan et al. (2009) and adopting, as an upper limit, the disc scale of $\approx 1$ arcsec determined from X-ray observation (Baganoff et al. 2001), we obtain $\mathscr{R} \approx 0.4 \%$, which indicates that the quadrupole moment measurement of Sgr A* would not be biased by the contribution of the disc above the $1 \%$ precision level. Furthermore, in a very resent publication, based on current X-ray and millimeter observations, Psaltis (2011) concludes that for compact orbits, like the ones discussed in this paper, hydrodynamic drag forces from plasma in the vicinity of Sgr $A^{*}$ are expected to be negligible.

\section{ACKNOWLEDGEMENTS}

We are grateful to K. J. Lee and G. X. Li for valuable discussions, and would like to thank J. P. W. Verbiest for carefully reading the paper and providing detailed comments. We also would like to thank the anonymous referee for his careful review of this manuscript, and for his useful comments. This research has made use of NASA's Astrophysics Data System Bibliographic Services. KL is funded by a stipend of the Max-Planck-Institute for Radio Astronomy.
Edwards, R. T., Hobbs, G. B., \& Manchester, R. N. 2006, MNRAS, 372, 1549

Eisenhauer, F., et al. 2009, in Science with the VLT in the ELT Era, ed. A. Moorwood, Vol. Astrophysics and Space Science Proceedings (Springer Netherlands), 361-365

Freire, P. C. C., et al. 2011, MNRAS, 412, 2763

Garfinkel, B. 1959, AJ, 64, 353

Genzel, R., Schödel, R., Ott, T., Eckart, A., Alexander, T., Lacombe, F., Rouan, D., \& Aschenbach, B. 2003, Nature, 425, 934

Ghez, A. M., et al. 2008, ApJ, 689, 1044

Gillessen, S., Eisenhauer, F., Trippe, S., Alexander, T., Genzel, R., Martins, F., \& Ott, T. 2009, ApJ, 692, 1075

Hansen, R. O. 1974, Journal of Mathematical Physics, 15, 46

Heusler, M. 1998, Living Reviews in Relativity 1, 6. URL (cited on 2011/7/22): http://www.livingreviews.org/lrr-1998-6

Hobbs, G., Lyne, A. G., \& Kramer, M. 2010, MNRAS, 402, 1027

Hobbs, G. B., Edwards, R. T., \& Manchester, R. N. 2006, MNRAS, 369, 655 
Jacoby, B. A., Hotan, A., Bailes, M., Ord, S., \& Kuklarni, S. R. 2005, ApJ, 629, L113

Johannsen, T., \& Psaltis, D. 2011, ApJ, 726, 11

Johnston, S., Kramer, M., Lorimer, D. R., Lyne, A. G., McLaughlin, M., Klein, B., \& Manchester, R. N. 2006, MNRAS, 373, L6

Keshet, U., Hopman, C., \& Alexander, T. 2009, ApJ, 698, L64

Kocsis, B., \& Tremaine, S. 2011, MNRAS, 412, 187

Königsdörffer, C., \& Gopakumar, A. 2005, Phys. Rev. D, 71, 024039

Kopeikin, S. M. 1997, Journal of Mathematical Physics, 38, 2587

Kramer, M. 1998, ApJ, 509, 856

Kramer, M., Backer, D. C., Cordes, J. M., Lazio, T. J. W., Stappers, B. W., \& Johnston, S. 2004, New Astron. Rev., 48, 993

Kramer, M., Klein, B., Lorimer, D. R., Müller, P., Jessner, A., \& Wielebinski, R. 2000, in ASP Conf. Ser. 202: IAU Colloq. 177: Pulsar Astronomy - 2000 and Beyond, ed. M. Kramer, N. Wex, \& R. Wielebinski, 37

Kramer, M., et al. 2006, Science, 314, 97

Kramer, T., \& Wex, N. 2009, Class. Quantum Grav., 26

Liu, K., Verbiest, J. P. W., Kramer, M., Stappers, B. W., van Straten, W., \& Cordes, J. M. 2011, MNRAS, 417, 2916

Löhmer, O., Kramer, M., Mitra, D., Lorimer, D. R., \& Lyne, A. G. 2001, ApJ, 562, L157

Lorimer, D. R., \& Kramer, M. 2005, Handbook of Pulsar Astronomy (Cambridge University Press)

Lyne, A., Hobbs, G., Kramer, M., Stairs, I., \& Stappers, B. 2010, Science, 329, 408

Macquart, J., Kanekar, N., Frail, D. A., \& Ransom, S. M. 2010, ApJ, 715, 939

Maron, O., Kijak, J., Kramer, M., \& Wielebinski, R. 2000, A\&AS, 147, 195

Melatos, A., \& Warszawski, L. 2009, ApJ, 700, 1524

Merritt, D., Alexander, T., Mikkola, S., \& Will, C. M. 2010, Phys. Rev. D, 81, 062002
O'Leary, R. M., Kocsis, B., \& Loeb, A. 2009, MNRAS, 395, 2127

Penrose, R. 1979, in General Relativity: An Einstein centenary survey, ed. S. W. Hawking \& W. Israel, Vol. 1 (Cambridge; New York: Cambridge University Press), 581-638

Pfahl, E., \& Loeb, A. 2004, ApJ, 615, 253

Price, R. H. 1972a, Phys. Rev. D, 5, 2419

—. 1972b, Phys. Rev. D, 5, 2439

Psaltis, D. 2008, Living Reviews in Relativity 11, 9. URL (cited on 2011/7/22: http://www.livingreviews.org/lrr-2008-9

—. 2011, arXiv:astro-ph/1112.0026

Pugh, G. E. 1959, WSEG research Memorandom No.11, Weapons Systems Evaluation Group, The Pentagon, Washington

Rickett, B. J. 1990, Ann. Rev. Astr. Ap., 28, 561

Robertson, H. P. 1938, Ann. Math., 38, 101

Sadeghian, L., \& Will, C. M. 2011, arXiv:astro-ph/1106.5056

Schiff, L. I. 1960, Physical Review Letters, 4, 215

Schödel, R., et al. 2002, Nature, 419, 694

Taylor, J. H. 1992, Philos. Trans. Roy. Soc. London A, 341, 117

Taylor, J. H. 1994, in Les Prix Nobel (Stockholm: Norstedts Tryckeri), 80-101

Vigeland, S. J., \& Hughes, S. A. 2010, Phys. Rev. D, 81, 024030

Wang, Y., Creighton, T., Price, R. H., \& Jenet, F. A. 2009a, ApJ, 705,1252

Wang, Y., Jenet, F. A., Creighton, T., \& Price, R. H. 2009b, ApJ, 697,237

Weisberg, J. M., Romani, R. W., \& Taylor, J. H. 1989, ApJ, 347, 1030

Wex, N. 1995, Class. Quantum Grav., 12, 983

—. 1998, MNRAS, 298, 67

Wex, N., \& Kopeikin, S. 1999, ApJ, 513, 388

Will, C. M. 2008, ApJ, 674, L25

Yuan, Y.-F., Cao, X., Huang, L., \& Shen, Z.-Q. 2009, ApJ, 699, 722

Zamaninasab, M., et al. 2011, MNRAS, 413, 322 